\title{
Local Allergic Inflammation in Chronic Rhinosinusitis With Nasal Polyps Could Influence on Disease Severity and Olfaction
}

\author{
Byung kee Youn, $\mathrm{MD}^{1 *}$, Dong-Kyu Kim, $\mathrm{MD}^{2 \star}$, Byung Hum Kim, MD', \\ Hyung Gu Kim, MD', Jin Hyeok Jeong, MD¹, and Seok Hyun Cho, MD \\ ${ }^{1}$ Department of Otorhinolaryngology-Head and Neck Surgery, Hanyang University College of Medicine, Seoul, Republic of Korea \\ ${ }^{2}$ Department of Otorhinolaryngology-Head and Neck Surgery, Chuncheon Sacred Heart Hospital, Hallym University College of Medicine, \\ Chuncheon, Republic of Korea
}

Background and Objectives: Chronic rhinosinusitis with nasal polyps (CRSwNP) is a multifactorial disease resulting from inflammation of the nasal cavity and paranasal sinuses. Systemic allergic inflammation is an important cause of CRSwNP; however, the effect of local allergic inflammation is unclear. This study was designed to investigate the effect of local allergic inflammation in CRSwNP.

Materials and Methods: The study included 11 patients with CRSwNP and 18 control subjects. Olfactory function was measured with the Korean Version of Sniffin's stick test. Nasal lavage fluids (NLFs) were collected from all subjects and analyzed for total IgE, eosinophilic cationic protein (ECP), and cytokines (tumor necrosis factor [TNF]- $\alpha$, interleukin [IL]-4, IL-10, IL-17A, interferon- $\gamma$ ). Flow cytometry was used to measure various inflammatory cells in the NAL fluids.

Results: On analysis of flow cytometry and enzyme-linked immunosorbent assay, we found that CRSwNP patients had significantly increased eosinophil (\%) and ECP levels in NLFs. In addition, there was significant local-systemic correlation between ECP level in NLFs and blood eosinophils (\%) ( $\mathrm{r}=0.391)$; however, there was no significant association between eosinophils (\%) in NLFs and blood eosinophils. Moreover, in CRSwNP patients, the severity of disease was related with blood eosinophil (\%), eosinophil (\%), and ECP levels in NLFs, whereas olfactory function was associated with blood eosinophil (\%) and ECP levels in NLFs.

Conclusion: CRSwNP is a disease with high allergic inflammation that has negative impacts on the severity of disease and olfactory function. Therefore, we suggest that control of local allergic inflammation will be helpful to treat CRSwNP patients.

Keywords: Chronic rhinosinusitis; Polyp; Local; Immune.

\section{INTRODUCTION}

Chronic rhinosinusitis (CRS) is defined as an inflammatory condition involving the paranasal sinuses, which persists for 12 weeks or longer. It is associated with two or more symptoms of either nasal obstruction or nasal discharge (anterior/posterior nasal drip), facial pain/pressure, and/or reduction/loss of smell. Currently, this disease classified as subgroups of patients based on nasal endoscopic findings, either

Received: May 21, 2021 Revised: June 28, 2021

Accepted: July 1, 2021

Address for correspondence: Seok Hyun Cho, MD, Department of Otorhinolaryngology-Head and Neck Surgery, Hanyang University College of Medicine, 222-1 Wangshimni-ro, Seongdong-gu, Seoul 04763, Republic of Korea Tel: +82-2-2290-8583, Fax: +82-2-2-2290-8588, E-mail: shcho@hanyang.ac.kr

*These authors contributed equally to this work.

This is an Open Access article distributed under the terms of the Creative Commons Attribution Non-Commercial License (https://creativecommons.org/licenses/bync/4.0) which permits unrestricted non-commercial use, distribution, and reproduction in any medium, provided the original work is properly cited. accompanied by nasal polyps (CRSwNP) or without nasal polyps (CRSsNP). ${ }^{1)}$ Generally, CRSsNP comprises more than two-thirds of cases and is less likely to be managed by surgical intervention, whereas CRSwNP represents $20 \%-25 \%$ of cases and frequently need surgical intervention. ${ }^{2)}$ The major pathogenesis of CRS is considered as alterations in mucociliary clearance, abnormalities in the sinonasal epithelial cell barrier, and tissue remodeling. ${ }^{2-4)}$ Additionally, the upregulated host innate and adaptive immune responses may be involved in its pathogenesis. ${ }^{2-4)}$ Specifically, most patients with CRSwNP have shown a predominant type 2 immune profile, compare to those with CRSsNP. ${ }^{5)}$ However, the exact pathogenesis of CRSwNP is still unclear.

Recently, the presence of local allergy in the nasal cavity was found in a number of patients diagnosed previously with non-allergic rhinitis. ${ }^{6-8)}$ Evidences for local allergy are supported by clinical symptoms, local production of allergen-specific immunoglobulin E (sIgE), a type 2 helper T cell inflammato- 
ry pattern in nasal secretions during natural exposure to aeroallergens, and a positive response to nasal allergen provocation with local nasal production of slgE to aeroallergens, tryptase, and eosinophil cationic protein (ECP). ${ }^{9)}$ However, to date, there was little known about the effect of local immune response on clinical relevance in patients with CRSwNP. Therefore, in the present study, we evaluated the local immunologic profile using nasal lavage fluids (NLFs) in CRSwNP patients and also investigated its clinical relevance, such as disease severity and olfactory function.

\section{MATERIALS AND METHODS}

\section{Subjects and sampling of nasal fluids}

From December 2015 to August 2016, we enrolled 29 consecutive patients. The diagnosis of CRS was based on personal medical history, physical examination, nasal endoscopy and CT findings of the nasal cavity with sinuses according to the 2012 European position paper on rhinosinusitis and nasal polyps (EPOS) guidelines. ${ }^{1)}$ Patient exclusion criteria were as follows: 1) younger than 18 years old; 2) previous treatment with antibiotics, systemic or topical corticosteroids, or other immune-modulating drugs up to 4 weeks before surgery; and 3) conditions such as unilateral rhinosinusitis, antrochoanal polyps, allergic fungal sinusitis, cystic fibrosis, or immotile ciliary disease. Samples from control subjects were obtained from patients with septal surgeries who do not have any sinonasal diseases. Paranasal CT images were staged with the LundMackay score for identifying CRS severity. Allergic rhinitis was diagnosed with a medical history and skin prick test. The diagnosis of asthma was based on the medical history and lung function analysis, including methacholine challenge test by pulmonologists. Patients were asked to complete the questionnaire reporting their symptoms (itching, sneezing, rhinorrhea, and nasal obstruction) at baseline and we calculated total nasal symptom score (TNSS). Quality of life (QOL) was measured on a 10-point visual analog scale. Additionally, all subjects underwent psychological olfactory test using the Korean version II of the Sniffin' Sticks Test and calculated the sum of Threshold-Discrimination-Identification (TDI). Moreover, we collected NLFs as previous described method under general anesthesia. ${ }^{10)}$ Briefly, under general anesthesia, we flushed each nostril with $5 \mathrm{~mL}$ of isotonic saline. The fluid was aspirated with a suction collector and then, immediately transferred to the laboratory bench. After the removal of crust and debris, the fluids were centrifuged at 6,000 rpm for $5 \mathrm{~min}$. After centrifuges, the cell pellets were used for the flow cytometry and the supernatants were analyzed by ImmunoCAP and enzyme-linked immunosorbent assay (ELISA). This study was approved by the Institutional Review Board of Hanyang
University Medical Center with a waiver of informed consent (IRB No. HYI 12-012).

\section{Flow cytometry}

We used the same antibody panel and gating strategy for the detection of nasal T cells (CD3), B cell (CD19), macrophages (CD14), dendritic cells (CD11c), and eosinophils (CD16) as previously described. ${ }^{10)}$ Flow cytometry was performed using a FACS Canto II (BD Biosciences, San Jose, CA, USA) and data were analyzed using Flow Jo ver. 9.3.2 (Treestar, Inc, San Carlos, CA, USA).

\section{ImmunoCAP and ELISA}

The concentrations of total IgE was measured using the ImmunoCAP $^{\circledR}$ assay (Phadia, Uppsala, Sweden). Immunologic profiled (ECP, tumor necrosis factor [TNF]- $\alpha$, interleukin [IL]-4, IL-10, IL-17A, and interferon [IFN]- $\gamma$ ) were also analyzed using commercially available ELISA kits according to the manufacture's recommendations.

\section{Statistical analysis}

Data were presented as medians with/without interquartile range and statistical significance $(\mathrm{p}<0.05)$ was analyzed using the Mann-Whitney U test. The relationships between two variables were examined using Spearman correlation analysis. All statistical analyses were performed using SPSS Statistics 21.0 (IBM Corp., Armonk, NY, USA).

\section{RESULTS}

\section{Clinical characteristics of subjects}

The clinical characteristics of each study group are summarized in Table 1. There was no difference in sex, allergic rhinitis, asthma, TNSS, QOL, and total IgE between the CRSwNP and controls. However, CRSwNP group showed significantly increased age, higher CT score, and eosinophils (\%) compared to control, whereas TDI score was lower in CRSwNP than in control.

\section{Local cellular and humoral immunologic profile in chronic rhinosinusitis with nasal polyp}

To evaluate the difference in local cellular response between two groups, we performed flow cytometry using NLFs. We found that there was no difference in innate immune cells (macrophages, dendritic cells, and neutrophils) and lymphocytes ( $\mathrm{T}$ cells and B cells) between control and CRSwNP. However, CRSwNP group had significantly increased local eosinophilia compared to control ( $\mathrm{p}=0.021)$ (Table 2).

Next line, to investigate the difference of local humoral response between two groups, we evaluated the immunologic 
profile of NLFs using immunoCAP and ELISA. There was no significant difference in Th1 (TNF- $\alpha$, IFN- $\gamma$ ), Th2 (IL-4, total IgE), Th17 (IL-17A), and Treg (IL-10) markers between two groups. However, the expression of ECP level was significantly increased in CRSwNP group, compared to control ( $\mathrm{p}=$ 0.037) (Table 3).

\section{Effect of local allergic inflammation on the clinical relevance of patients with chronic rhinosinusitis with nasal polyp}

On analysis of the correlation between local and systemic allergic inflammation, we found a significant local-systemic correlation between ECP level in NLFs and blood eosinophil (\%) $(r=0.391)$ (Fig. 1). However, there was no significant association for eosinophil (\%) in NLFs with blood eosinophil (\%) or ECP level in NLFs.

In addition, we assessed the clinical relevance of local or systemic allergic inflammation, such as disease severity and olfactory function (Fig. 2). We observed a significant correlation of blood eosinophil with disease severity and olfactory function, whereas eosinophil (\%) in NLFs showed an only significant relationship on disease severity, not olfactory function. However, ECP level in NLFs showed a significant correlation with disease severity and olfactory function $(\mathrm{r}=0.387$ and $\mathrm{r}=-0.415$, respectively)

\section{DISCUSSION}

Numerous studies have supported that systemic allergic inflammation may be an important cause of development of CRSwNP. ${ }^{23) 11112)}$ However, studies for local allergic inflammation on CRSwNP are still limited. ${ }^{13-15)}$ Thus, in the present study, we evaluated the local allergic inflammation status and analyzed its clinical relevance in patients with CRSwNP. Our major findings can be summarized as follows: 1) CRSwNP

Table 3. Comparison of local humoral immune responses between control and chronic rhinosinusitis with nasal polyps (CRSwNP)

\begin{tabular}{lccc}
\multicolumn{1}{c}{ Variable } & \multicolumn{1}{c}{ Control } & CRSwNP & p value \\
\hline ECP $(\mu \mathrm{g} / \mathrm{L})$ & $18.9(13.5-65.7)$ & $71.2(42.1-143.8)$ & $0.037^{*}$ \\
TNF- $\alpha(\mathrm{pg} / \mathrm{mL})$ & $1.5(0.0-4.1)$ & $4.3(2.5-9.9)$ & 0.176 \\
IL-4 $(\mathrm{pg} / \mathrm{mL})$ & $2.4(0.0-6.3)$ & $0.0(0.0-5.7)$ & 0.275 \\
IL-10 $(\mathrm{pg} / \mathrm{mL})$ & $0.0(0.0-0.0)$ & $0.0(0.0-5.1)$ & 0.298 \\
IL-17A $(\mathrm{pg} / \mathrm{mL})$ & $0.0(0.0-0.0)$ & $0.0(0.0-1.21)$ & 0.940 \\
IFN- $\gamma(\mathrm{pg} / \mathrm{mL})$ & $8.4(2.1-15.7)$ & $27.5(1.1-32.9)$ & 0.433 \\
Total IgE $(\mathrm{kU} / \mathrm{L})$ & $5.5(4.7-9.7)$ & $5.3(4.0-9.4)$ & 0.774 \\
\hline
\end{tabular}

${ }^{*} \mathrm{p}<0.05$. ECP, eosinophilic cationic protein; IL, interleukin; IFN, interferon; IgE, immunoglobulin $\mathrm{E}$

Table 1. Clinical characteristic of the subjects

\begin{tabular}{lccc}
\hline \multicolumn{1}{c}{ Characteristic } & Control $(\mathrm{n}=18)$ & CRSwNP $(\mathrm{n}=11)$ & $\mathrm{p}$ value \\
\hline Sex $(\mathrm{M}: \mathrm{F})$ & $15: 3$ & $9: 2$ & 0.644 \\
Age (year) & $23.5(19.8-33.5)$ & $49.0(26.0-66.0)$ & $0.035^{*}$ \\
Allergic rhinitis (\%) & 66.7 & 45.5 & 0.230 \\
Asthma (\%) & 0 & 9.1 & 0.379 \\
TNSS & $7.0(5.5-9.0)$ & $5.0(4.0-8.0)$ & 0.191 \\
QOL & $4.0(2.0-4.0)$ & $3.0(1.0-4.0)$ & 0.578 \\
TDI & $36.0(32.5-36.5)$ & $27.0(12.0-32.0)$ & $0.002^{*}$ \\
CT score & $0.0(0.0-0.0)$ & $15.0(12.5-16.0)$ & $0.001^{* *}$ \\
Total IgE & $108.2(39.2-332.8)$ & $130.7(117.4-200.0)$ & 0.643 \\
Eosinophils $(\%)$ & $2.6(1.2-3.9)$ & $4.5(2.7-6.2)$ & $0.039^{*}$ \\
\hline
\end{tabular}

${ }^{*} \mathrm{p}<0.05 ;{ }^{* *} \mathrm{p}<0.01$. CRSwNP, chronic rhinosinusitis with nasal polyps; TNSS, total nasal symptom score; QOL, quality of life; TDI, Threshold-Discrimination-Identification; IgE, immunoglobulin E

Table 2. Comparison of local cellular immune responses between control and chronic rhinosinusitis with nasal polyps (CRSwNP)

\begin{tabular}{lccc}
\hline \multicolumn{1}{c}{ Variable } & Control & CRSwNP & p value \\
\hline WBC count $\left(/ \mathrm{mm}^{3}\right)$ & $14,160(8,349-22,925)$ & $100,199(10,960-215,799)$ & 0.084 \\
Macrophages (\%) & $0.55(0.31-1.61)$ & $1.20(0.38-1.54)$ & 0.492 \\
Dendritic cells (\%) & $1.02(0.42-1.34)$ & $0.28(0.21-0.53)$ & 0.076 \\
T cells (\%) & $18.86(5.38-34.10)$ & $5.83(3.97-12.54)$ & 0.102 \\
B cells (\%) & $4.75(1.17-8.89)$ & $1.87(0.53-3.50)$ & 0.076 \\
Neutrophils (\%) & $67.08(32.28-70.45)$ & $44.54(26.31-60.61)$ & 0.298 \\
Eosinophils (\%) & $3.53(1.98-10.03)$ & $10.28(7.87-24.71)$ & $0.021^{*}$ \\
\hline
\end{tabular}

${ }^{*} \mathrm{p}<0.05$ 
patients showed an increased local allergic inflammation, such as eosinophils (\%) and ECP level, compared to controls. 2) Among local allergic inflammation, ECP level in nasal secretion was a significantly associated with blood eosinophil (\%), but there was no relationship between eosinophil (\%) in blood and nasal secretion. 3) Interestingly, we found that disease severity and olfactory function in patients with CRSwNP were well reflected by blood eosinophil (\%) and ECP level in nasal secretion.

Eosinophils are responsible for tissue damage and inflammation in many diseases, specifically in allergic disease. It is known that several mediators derived from eosinophils can damage epithelial cells, stimulate epithelial-to-mesenchymal transition, and alter mechanical responses of airways. ${ }^{16-19)}$ To date, correlations between disease severity and eosinophils measured in blood or nasal tissues are well established. ${ }^{15) 20-23)}$ These studies described that eosinophilia correlates strongly with type 2 immune and inflammatory responses, and eosinophilic CRSwNP patients showed a higher prevalence and severity of asthma. Additionally, one recent large scale study, ${ }^{24)}$ the Japanese Epidemiological Survey of Refractory Eosinophilic Chronic Rhinosinusitis study which evaluated eosino- phils in the blood and tissue, suggested that blood eosinophils, ethmoid disease, asthma, and aspirin intolerance are well associated with the recurrence rate of CRS patients. Consistent with these reports, eosinophilia in blood and nasal secretion was significantly associated with disease severity in this study, and we also observed the positive relationship between ECP level in nasal secretion and disease severity. Moreover, several studies demonstrated that olfactory dysfunction in CRS patients correlates local eosinophilia. ${ }^{2526)}$ Although we could not find a significant relationship between eosinophilia in nasal secretion and olfactory function, ECP level in nasal secretion had negatively correlated with olfactory function. Together, these data indicated that ECP level in nasal secretion as a marker for local allergic inflammation is more wellreflected the clinical relevance in patients with CRSwNP, compare to eosinophilia in nasal secretion.

However, our study has some limitations. First, in this study, we could not evaluate tissue eosinophilia, because tissue biopsies were not performed. Second, CRSwNP generally has two endotypes, such as eosinophilic and non-eosinophilic types. Eosinophilic CRSwNP is characterized by increased type 2 immune response with tissue eosinophilia, whereas non-eo-

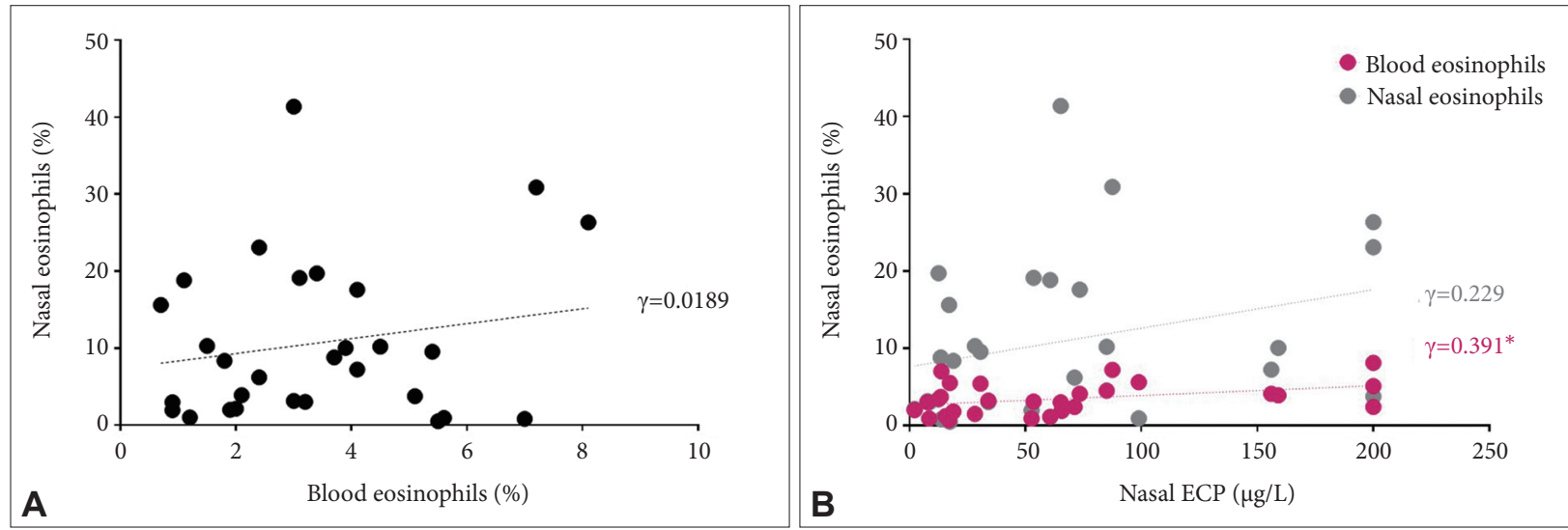

Fig. 1. Evaluation of interaction local to systemic allergic inflammation $\left({ }^{*} p<0.05\right)$. A: Relationship between blood and nasal eosinophils (\%). B: Realtionship between nasal ECP and eosinophils (\%). ECP, eosinophil cationic protein.
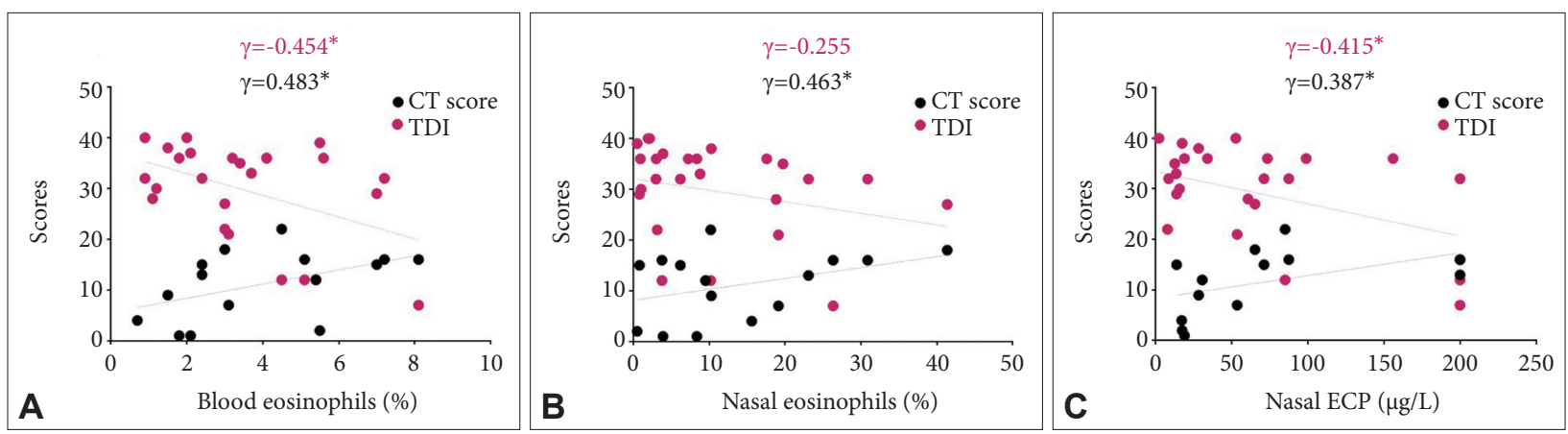

Fig. 2. Effect of local allergic inflammation on clinical relevance. A: Relationship between blood eosinophils (\%) and CT score/olfactory function (TDI). B: Relationship between nasal eosinophils (\%) and CT score/olfactory function (TDI). C: Relationship between nasal ECP level and CT score/olfactory function (TDI). ${ }^{*} p<0.05$. TDI, Threshold-Discrimination-Identification. ECP, eosinophil cationic protein. 
sinophilic CRSwNP has a mixed immune response with predominant tissue infiltration of neutrophils and lymphocytes. Thus, the effect of local allergic inflammation may be different between each endotypes, but we could not differentiate the endotypes due to lack of nasal tissues obtained from patients. Third, this study showed a different mean age between the two groups. This difference may affect our outcomes as a confounding factor because the immune system undergoes age-related changes, including a gradual increase in the production and circulation of proinflammatory cytokines.

In conclusion, the elevated local allergic inflammation is detected in patients with CRSwNP. Eosinophilia and increased ECP level in nasal secretion are a signature marker for local allergic inflammation in CRSwNP patients. In addition, Eosinophilia and ECP level in nasal secretion is correlated with disease severity. Moreover, olfactory dysfunction correlates with ECP level in nasal secretion. Therefore, we suggest that clinicians have an interest in the control of local allergic inflammation when those treat CRSwNP patients.

\section{Conflicts of Interest \\ The authors have no potential conflicts of interest to disclose.}

\section{Author Contributions}

Conceptualization: Byung kee Youn, Seok Hyun Cho. Data curation: Byung kee Youn, Byung Hum Kim, Seok Hyun Cho. Formal analysis: Byung kee Youn, Byung Hum Kim. Funding acquisition: Seok Hyun Cho. Investigation: Byung kee Youn, Dong-Kyu Kim. Methodology: Byung kee Youn, Seok Hyun Cho. Project administration: Byung kee Youn. Resources: Byung kee Youn, Dong-Kyu Kim. Software: Dong-Kyu Kim. Supervision: Dong-Kyu Kim, Jin Hyeok Jeong. Validation: Hyung Gu Kim, Jin Hyeok Jeong. Visualization: Byung kee Youn, Hyung Gu Kim, Seok Hyun Cho. Writing-original draft: Dong-Kyu Kim, Seok Hyun Cho. Writing-review \& editing: Dong-Kyu Kim.

\section{ORCID iD}

Seok Hyun Cho https://orcid.org/0000-0001-8218-5894

\section{Funding Statement}

This work was supported by the National Research Foundation of Korea (NRF) grant funded by the Korea government (MSIP; Ministry of Science, ICT \& Future Planning) (No. 2017R1D1A1B03028797) and the research fund of Hanyang University (HY-2015).

\section{REFERENCES}

1) Fokkens WJ, Lund VJ, Mullol J, Bachert C, Alobid I, Baroody F, et al. EPOS 2012: European position paper on rhinosinusitis and nasal polyps 2012. A summary for otorhinolaryngologists. Rhinology 2012; 50(1):1-12.

2) Schleimer RP. Immunopathogenesis of chronic rhinosinusitis and nasal polyposis. Annu Rev Pathol 2017;12:331-57.

3) Stevens WW, Lee RJ, Schleimer RP, Cohen NA. Chronic rhinosinusitis pathogenesis. J Allergy Clin Immunol 2015;136(6):1442-53.

4) Tomassen P, Vandeplas G, Van Zele T, Cardell LO, Arebro J, Olze H, et al. Inflammatory endotypes of chronic rhinosinusitis based on cluster analysis of biomarkers. J Allergy Clin Immunol 2016;137(5): 1449-56.e4.

5) Bachert C, Wagenmann M, Hauser U, Rudack C. IL-5 synthesis is upregulated in human nasal polyp tissue.J Allergy Clin Immunol 1997; 99(6 Pt 1):837-42.

6) Rondón C, Romero JJ, López S, Antúnez C, Martín-Casañez E, Torres MJ, et al. Local IgE production and positive nasal provocation test in patients with persistent nonallergic rhinitis. J Allergy Clin Immunol 2007;119(4):899-905.

7) Wierzbicki DA, Majmundar AR, Schull DE, Khan DA. Multiallergen nasal challenges in nonallergic rhinitis. Ann Allergy Asthma Immunol 2008;100(6):533-7.

8) Rondón C, Canto G, Blanca M. Local allergic rhinitis: a new entity, characterization and further studies. Curr Opin Allergy Clin Immunol 2010; 10(1):1-7.

9) Rondón C, Fernandez J, Canto G, Blanca M. Local allergic rhinitis: concept, clinical manifestations, and diagnostic approach. J Investig Allergol Clin Immunol 2010;20(5):364-71.

10) Lee KS, Yu J, Shim D, Choi H, Jang MY, Kim KR, et al. Local immune responses in children and adults with allergic and nonallergic rhinitis. PLoS One 2016;11(6):e0156979.

11) Bachert C, Zhang N, Hellings PW, Bousquet J. Endotype-driven care pathways in patients with chronic rhinosinusitis. J Allergy Clin Immunol 2018;141(5):1543-51.

12) Kim DK, Eun KM, Kim MK, Cho D, Han SA, Han SY, et al. Comparison between signature cytokines of nasal tissues in subtypes of chronic rhinosinusitis. Allergy Asthma Immunol Res 2019;11(2):201-11.

13) Cheng KJ, Xu YY, Zhou ML, Zhou SH, Wang SQ. Role of local allergic inflammation and Staphylococcus aureus enterotoxins in Chinese patients with chronic rhinosinusitis with nasal polyps. J Laryngol Otol 2017;131(8):707-13.

14) Vagic D, Ferencic Z, Drvis P, Geber G, Dzidic S, Baudoin T, et al. Local IgE and inflammation in chronic rhinosinusitis of asthmatics and non-asthmatics. Eur Arch Otorhinolaryngol 2008;265(10):1205-9.

15) Cao PP, Zhang YN, Liao B, Ma J, Wang BF, Wang H, et al. Increased local IgE production induced by common aeroallergens and phenotypic alteration of mast cells in Chinese eosinophilic, but not non-eosinophilic, chronic rhinosinusitis with nasal polyps. Clin Exp Allergy 2014;44(5):690-700.

16) George L, Brightling CE. Eosinophilic airway inflammation: role in asthma and chronic obstructive pulmonary disease. Ther Adv Chronic Dis 2016;7(1):34-51.

17) Lee JJ, Jacobsen EA, McGarry MP, Schleimer RP, Lee NA. Eosinophils in health and disease: the LIAR hypothesis. Clin Exp Allergy 2010; 40(4):563-75.

18) Lee M, Kim DW, Yoon H, So D, Khalmuratova R, Rhee CS, et al. Sirtuin 1 attenuates nasal polypogenesis by suppressing epithelial-tomesenchymal transition. J Allergy Clin Immunol 2016;137(1):87-98.e7.

19) Shin HW, Cho K, Kim DW, Han DH, Khalmuratova R, Kim SW, et al. Hypoxia-inducible factor 1 mediates nasal polypogenesis by inducing epithelial-to-mesenchymal transition. Am J Respir Crit Care Med 2012;185(9):944-54.

20) Szucs E, Ravandi S, Goossens A, Beel M, Clement PA. Eosinophilia in the ethmoid mucosa and its relationship to the severity of inflammation in chronic rhinosinusitis. Am J Rhinol 2002;16(3):131-4.

21) Nakayama T, Yoshikawa M, Asaka D, Okushi T, Matsuwaki Y, Otori $\mathrm{N}$, et al. Mucosal eosinophilia and recurrence of nasal polyps-new classification of chronic rhinosinusitis. Rhinology 2011;49(4):392-6.

22) McHugh T, Snidvongs K, Xie M, Banglawala S, Sommer D. High tissue eosinophilia as a marker to predict recurrence for eosinophilic chronic rhinosinusitis: a systematic review and meta-analysis. Int Forum Allergy Rhinol 2018;8(12):1421-9.

23) Bryson JM, Tasca RA, Rowe-Jones JM. Local and systemic eosinophilia in patients undergoing endoscopic sinus surgery for chronic rhino- 
sinusitis with and without polyposis. Clin Otolaryngol Allied Sci 2003; 28(1):55-8.

24) Tokunaga $T$, Sakashita $M$, Haruna $T$, Asaka D, Takeno S, Ikeda H, et al. Novel scoring system and algorithm for classifying chronic rhinosinusitis: the JESREC Study. Allergy 2015;70(8):995-1003.

25) Lavin J, Min JY, Lidder AK, Huang JH, Kato A, Lam K, et al. Superior turbinate eosinophilia correlates with olfactory deficit in chronic rhinosinusitis patients. Laryngoscope 2017;127(10):2210-8.

26) Hauser LJ, Chandra RK, Li P, Turner JH. Role of tissue eosinophils in chronic rhinosinusitis-associated olfactory loss. Int Forum Allergy Rhinol 2017;7(10):957-62. 